\title{
Separate- versus common-common-cause-type derivations of the Bell inequalities
}

\author{
Gábor Hofer-Szabó \\ Department of Philosophy and History of Science \\ Budapest University of Technology and Economics \\ Email:gszabo@hps.elte.hu
}

\begin{abstract}
Standard derivations of the Bell inequalities assume a common common cause system that is a common screener-off for all correlations and some additional assumptions concerning locality and no-conspiracy. In a recent paper (Graßhoff et al., 2005) Bell inequalities have been derived via separate common causes assuming perfect correlations between the events. In the paper it will be shown that the assumptions of this separate-common-cause-type derivation of the Bell inequalities in the case of perfect correlations can be reduced to the assumptions of a commoncommon-cause-system-type derivation. However, in the case of non-perfect correlations a nonreducible separate-common-cause-type derivation of some Bell-like inequalities can be given. The violation of these Bell-like inequalities proves Szabó's (2000) conjecture concerning the nonexistence of a local, non-conspiratorial, separate-common-cause-model for a $\delta$-neighborhood of perfect EPR correlations.
\end{abstract}

\section{Introduction}

In a recent paper Graßhoff, Portmann, and Wüthrich (2005) presented a new derivation of the Bell inequalities using weaker premises than standard derivations do. Standard derivations of the Bell inequalities assume (beside other premises) a so-called common common cause system that is a common screener-off for all correlations. Graßhoff et al. derived Bell inequalities instead via separate common causes that is different screener-offs for the different correlations. Since the historical steps that led to the problem are of some interest we briefly sketch the preliminaries of this result.

The conceptual difference between common common cause and separate common cause was first recognized by Belnap and Szabó (1996). Belnap and Szabó pointed out that in standard commoncausal explanations of the EPR correlations common cause is actually meant as a common common cause accounting for all correlations. Therefore, in their analysis of the GHZ experiment Belnap and Szabó applied a weaker concept, namely a system of separate common causes explaining the correlations separately.

Concerning the algebraic-probabilistic features of the Reichenbachian common cause Hofer-Szabó, Rédei, and Szabó (1999) proved the following proposition. Classical (and also non-classical) correlations can be given a probabilistic common-causal explanation in the sense that any classical (and non-classical) probability measure space with correlating pairs of events can be extended such that the extension contains a Reichenbachian separate screener-off for each correlation. (For the precise definitions see below.) Same authors proved in (2002) that this proposition does not apply if Reichenbachian separate screener-offs are replaced with Reichenbachian common screener-offs. In other words, classical probability measure spaces containing correlating pairs of events generally cannot be extended such that the extension contains a Reichenbachian common screener-off for all correlations. 
Thus, being a common common cause of a set of correlations turned out to be a much stronger demand than being a common cause of a single correlation. Finally, Hofer-Szabó and Rédei (2004, 2006) generalized the notion of the Reichenbachian common cause for situations when the correlation is due to a system of different causes. This new concept was called Reichenbachian common cause system. Propositions concerning the difference between Reichenbachian common cause systems and Reichenbachian common common cause systems were proven similarly.

Szabó (2000) applied the concept of separate common cause for the EPR situation. Since factorizability, locality and no-conspiracy together entail various types of Bell inequalities, EPR correlations cannot be given a local, non-conspiratorial, common-common-cause-system-model. Now, Szabó's idea was to replace the common common cause system with separate common causes and thus in avoiding Bell inequalities to give a separate-common-cause-model for the EPR correlations. He constructed a number of separate-common-cause-models which were local and non-conspiratorial in the usual sense that the measurement settings were statistically independent from the different common causes. However, the models were conspiratorial on a deeper level. The measurement settings statistically correlated with various algebraic combinations of the separate common causes. After numerous computer simulations aiming to remove the unwanted conspiracies Szabó concluded with the conjecture in the Appendix of (2000) that EPR can not be given any local, non-conspiratorial, separate-common-cause-model.

A talk given by Miklós Rédei in Bern in March 2000 prompted Graßhoff, Portmann, and Wüthrich to investigate the possibility of separate common causes. In the course of their project they realized that Szabó's conjecture can be proved by deriving the Bell inequalities from separate common causes, locality and no-conspiracy. This result has been published in (Graßhoff et al., 2005).

A crucial premise of the derivation of the Bell inequalities given by Graßhoff et al. was that the correlation between some events had to be perfect. In our paper it will be shown that in the perfect correlation case the assumptions of the separate-common-cause-derivation of the Bell inequalities by Graßhoff et al. are reducible to a common screener-off system. In other words, the assumptions that are necessary for the derivation of the Bell inequalities in (Graßhoff et al., 2005) together entail the assumptions of the usual common-common-cause-system-derivation. However, assuming non-perfectly correlating pairs of events this entailment does not apply and hence a non-reducible separate-common-cause-type derivation of the Bell inequalities can be given. We will present this derivation in Section 7. Moreover, our derivation of the Bell inequalities in the non-perfect case excludes a local, non-conspiratorial, separate-common-cause-model not only for perfect EPR correlations but for any $\operatorname{EPR}(\delta)$ set that is for any set of correlations lying in a $\delta$-neighborhood of perfect EPR correlations.

In Section 2 we make explicit the concepts and propositions introduced informally in the Introduction. In Section 3 the standard common-common-cause-system-type derivation of the Bell inequalities will be recalled. In Section 4 we recapitulate Szabó's separate-common-cause-models for the EPR and his conjecture. In Section 5 the separate-common-cause-type derivation of the Bell inequalities by Graßhoff et al. will be presented. In Section 6 it will be pointed out that the assumptions of Graßhoff's separate-common-cause-type derivation of the Bell inequalities entail a common-screener-off-system. Finally, in Section 7 a non-reducible separate-common-cause-type derivation of the Bell inequalities will be presented.

\section{The variety of common causes}

Literature provides various terms for the notion of common cause, such as Reichenbachian common cause (Reichenbach, 1956; Hofer-Szabó, Rédei, Szabó, 1999), single common cause (Belnap, Szabó, 1996), multiple common cause (Placek, 2000), common common cause (Hofer-Szabó, Rédei, Szabó, 2002), common cause variable (Wüthrich, 2004; Graßhoff et al., 2005), and common cause system (Hofer-Szabó, Rédei, 2004, 2006). Therefore, we first lay down our terminology in a series of def- 
initions. Since the algebraic-probabilistic characterization yields only necessary but not sufficient conditions for an event to be a common cause, in the following definitions we will discern the term 'common cause' on the one hand and the term 'screener-off' on the other hand. Any event satisfying the defining conditions of the definitions will be referred to as a 'screener-off', whereas the same conditions will be only necessary but not sufficient conditions for an event to be a common cause.

We begin with Reichenbach's original definition of the common cause (Reichenbach, 1956). Let $(\Omega, p)$ be a classical probability measure space and let $A, B \in \Omega$ be two positively correlating events, i.e.

$$
p(A B)>p(A) p(B)
$$

Reichenbach characterizes the common cause of the correlation by the following necessary conditions:

Definition 1. An event $C$ in $\Omega$ is said to be the Reichenbachian common cause of the correlation between $A$ and $B$ only if the events $A, B$ and $C$ satisfy the following relations:

$$
\begin{aligned}
p(A B \mid C) & =p(A \mid C) p(B \mid C) \\
p(A B \mid \bar{C}) & =p(A \mid \bar{C}) p(B \mid \bar{C}) \\
p(A \mid C) & >p(A \mid \bar{C}) \\
p(B \mid C) & >p(B \mid \bar{C})
\end{aligned}
$$

where $\bar{C}$ denotes the complement of $C$ and $p(X \mid Y)=p(X Y) / p(Y)$ denotes the conditional probability of $X$ on condition $Y$. Equations (2)-(3) are called "screening-off" properties since conditioning on $C$ and $\bar{C}$, respectively screens off the correlation between $A$ and $B$. Inequalities (4)-(5) express the "positive statistical relevance" of the cause $C$ on the two effects $A$ and $B$, respectively. To stress the fact that conditions (2)-(5) are only necessary but not sufficient conditions for an event $C$ to be a Reichenbachian common cause, we refer to any event $C$ in $\Omega$ satisfying (2)-(5) as a Reichenbachian screener-off of the correlation between $A$ and $B$.

Now, what is a Reichenbachian common common cause? Let $(\Omega, p)$ be a classical probability measure space as before and let $\left(A_{1}, B_{1}\right)$ and $\left(A_{2}, B_{2}\right)$, respectively be two positively correlating pairs of events in $\Omega$, i.e. for $i=1,2$

$$
p\left(A_{i} B_{i}\right)>p\left(A_{i}\right) p\left(B_{i}\right)
$$

Then the Reichenbachian common common cause of both correlations is defined as follows:

Definition 2. An event $C$ in $\Omega$ is said to be the Reichenbachian common common cause of correlations $\left(A_{1}, B_{1}\right)$ and $\left(A_{2}, B_{2}\right)$, respectively only if for $i=1,2$ the following relations are satisfied:

$$
\begin{aligned}
p\left(A_{i} B_{i} \mid C\right) & =p\left(A_{i} \mid C\right) p\left(B_{i} \mid C\right) \\
p\left(A_{i} B_{i} \mid \bar{C}\right) & =p\left(A_{i} \mid \bar{C}\right) p\left(B_{i} \mid \bar{C}\right) \\
p\left(A_{i} \mid C\right) & >p\left(A_{i} \mid \bar{C}\right) \\
p\left(B_{i} \mid C\right) & >p\left(B_{i} \mid \bar{C}\right)
\end{aligned}
$$

Again, any event $C$ in $\Omega$ satisfying (7)-(10) for the correlations $\left(A_{1}, B_{1}\right)$ and $\left(A_{2}, B_{2}\right)$, respectively is called a Reichenbachian common screener-off of both correlations.

While the definition of the Reichenbachian common cause refers to a single correlating pair, the definition of the Reichenbachian common common cause relates to two (or more) correlations. This later definition requires much more from $C$, as it is shown in the following two propositions: 
Proposition 1. [Reichenbachian screener-off extendability] Let $(\Omega, p)$ be a classical probability measure space and let $(A, B)$ be a correlating pair in $\Omega$. Then there is an $\left(\Omega^{\prime}, p^{\prime}\right)$ extension of $(\Omega, p)$ such that for the correlation $(A, B)$ there exists a Reichenbachian screener-off $C$ in $\left(\Omega^{\prime}, p^{\prime}\right)$. (Hofer-Szabó, Rédei, Szabó, 1999)

Proposition 2. [No Reichenbachian common screener-off extendability] There exists an $(\Omega, p)$ classical probability measure space and two correlating pairs $\left(A_{1}, B_{1}\right)$ and $\left(A_{2}, B_{2}\right)$, respectively in $\Omega$ such that there is no $\left(\Omega^{\prime}, p^{\prime}\right)$ extension of $(\Omega, p)$ which contains a Reichenbachian common screener-off $C$ in $\left(\Omega^{\prime}, p^{\prime}\right)$ for both correlations. (Hofer-Szabó, Rédei, Szabó, 2000)

Proposition 1 claims that for a single correlating pair a common-cause-type explanation is always possible by extending the probability measure space in an appropriate way. (Moreover, if $\Omega$ contains $n \in \mathbb{N}$ correlating pairs, each correlation can be given a Reichenbachian-separate-common-causetype explanation in the sense that we apply the above extension $n$-times in turn for $(\Omega, p)$ such that the extended $\Omega^{(n)}$ contains a Reichenbachian separate screener-off for each correlation.) However, according to Proposition 2 this strategy does not work generally if we are going to obtain the same Reichenbachian common cause for the two (or more) correlating pairs. Thus, being a Reichenbachian common common cause imposes much stronger demand on $C$ than simply being a Reichenbachian common cause.

What is the situation if the correlation is due not only to a single causal factor $C$ but to a system of different causes? In other words, how can the notion of the Reichenbachian common cause be generalized for situations when more causes are present? This idea is spelled out in the next definition.

Definition 3. Let $(\Omega, p)$ be a classical probability measure space and let $A$ and $B$ be two correlating events in $\Omega$. A partition $\left\{C_{i}\right\}_{i \in I}$ of $\Omega$ is said to be the Reichenbachian common cause system for the correlation $(A, B)$ only if for all $i, j \in I(i \neq j)$ the following two conditions are satisfied:

$$
\begin{array}{r}
p\left(A B \mid C_{i}\right)=p\left(A \mid C_{i}\right) p\left(B \mid C_{i}\right) \\
\left(p\left(A \mid C_{i}\right)-p\left(A \mid C_{j}\right)\right)\left(p\left(B \mid C_{i}\right)-p\left(B \mid C_{j}\right)\right)>0
\end{array}
$$

We refer to an event $C$ in $\Omega$ satisfying (11)-(12) as a Reichenbachian screener-off system of the correlation.

The above definition is a natural generalization of Reichenbach's original definition of the common cause to the case when more than one single factor contribute to the correlation. Every element of the partition screens the correlations off and any two elements in the partition behave like a Reichenbachian common cause and its complement. The cardinality of the index set $I$ (i.e. the number of events in the partition) is called the size of the Reichenbachian common cause system. It is straightforward to see that the partition $\{C, \bar{C}\}$ generated by the Reichenbachian common cause $C$ is a Reichenbachian common cause system of size 2 . To stress this fact we will use the term 'Reichenbachian common cause' also for the partition $\{C, \bar{C}\}$.

However, in the standard EPR-Bell literature common cause of a correlation is used in a different way, purely as a factorizing partition of the correlation. To be in tune with this usage of the term the notion of common cause and common cause system will be defined as follows:

Let $(\Omega, p)$ be a classical probability measure space and let $(A, B)$ be a correlating pair of events in $\Omega$.

Definition 4. An event $C$ in $\Omega$ is said to be the common cause of the correlation only if the following relations hold:

$$
\begin{aligned}
& p(A B \mid C)=p(A \mid C) p(B \mid C) \\
& p(A B \mid \bar{C})=p(A \mid \bar{C}) p(B \mid \bar{C})
\end{aligned}
$$


Definition 5. A partition $\left\{C_{i}\right\}_{i \in I}$ of $\Omega$ is said to be the common cause system of the pair $(A, B)$ only if for all $i \in I$ the following conditions are satisfied:

$$
p\left(A B \mid C_{i}\right)=p\left(A \mid C_{i}\right) p\left(B \mid C_{i}\right)
$$

Finally, an event $C$ in $\Omega$ satisfying (13)-(14) is called a screener-off , and a partition $\left\{C_{i}\right\}_{i \in I}$ of $\Omega$ satisfying (15) is called a screener-off system of the correlation $(A, B)$.

It is obvious that the definition of the Reichenbachian common cause turns into that of the common cause if we drop inequalities (4)-(5), and in the same manner, the definition of the Reichenbachian common cause system leads to that of the common cause system if we drop inequality (12). Thus, we obtain four different common-causal definitions, namely Reichenbachian common cause, Reichenbachian common cause system, common cause, and common cause system, respectively. It can be seen easily in the appropriate definition that Reichenbachian common cause is the strongest of the four notions and common cause system is the weakest.

Again, it must be obvious how the definitions of the appropriate common common cause, common common cause system, and Reichenbachian common common cause system, respectively arise if we take not one but two correlating pairs $\left(A_{1}, B_{1}\right)$ and $\left(A_{2}, B_{2}\right)$, respectively.

In Proposition 1 it was claimed that for any correlating pair $(A, B)$ in a classical probability measure space $(\Omega, p)$ the algebra $\Omega$ can be extended in such a way that there exists a Reichenbachian screener-off for the correlation in the extended algebra. Since the definition of the Reichenbachian common cause is stronger than that of the other three types of common cause, Proposition 1 remains valid if we replace Reichenbachian screener-off with screener-off, screener-off system and Reichenbachian screener-off system, respectively. Thus, classical correlations can be given a common-causeexplanation in any of the four different ways.

However, Proposition 2 claimed that no Reichenbachian-common-common-cause-explanation can be generally provided for two different correlations in a classical probability measure space by extending the algebra. Again, since the definition of the Reichenbachian common cause is stronger than that of the other three notions, extendability can hold for the weaker cases. And indeed, this is the case. For the case of the common cause system the following proposition holds:

Proposition 3. Let $(\Omega, p)$ be a classical probability measure space and let $\left(A_{1}, B_{1}\right)$ and $\left(A_{2}, B_{2}\right)$, respectively be two arbitrary correlating pairs in $\Omega$. Then the partition generated by the events $A_{1}$, $A_{2}, B_{1}$ and $B_{2}$ yields a common screener-off system in $\Omega$ for both correlations.

Obviously, the partition generated by the correlating events screens off the correlations between the pairs $\left(A_{1}, B_{1}\right)$ and $\left(A_{2}, B_{2}\right)$, respectively and hence it trivially regards as a common screener-off system. Furthermore, this common screener-off system takes place in the original algebra and hence there is no need to extend $\Omega$. For these reasons the common screener-off system due to this partition is not a physically sound common-causal explanation of the correlations.

\section{Common common cause system and Bell inequalities}

Consider the usual EPR experimental setup with a pair of spin- $\frac{1}{2}$ particles prepared in the singlet state $\left|\Psi_{s}\right\rangle$. Let $p\left(a_{i}\right)$ denote the probability that the spin measurement apparatus is set to measure the spin in direction $i \quad(i=1,2,3)$ in the left wing and let $p\left(b_{j}\right)$ denote the same for direction $j \quad(j=1,2,3)$ in the right wing. Furthermore, let $p\left(A_{i}\right)$ stand for the probability that the spin measurement in direction $i$ in the left wing yields the result +1 and let $p\left(\bar{A}_{i}\right)$ denote the probability that the measurement in direction $i$ in the left wing yields the result -1 . Let $p\left(B_{j}\right)$ and $p\left(\bar{B}_{j}\right)$ be defined in a similar way in the right wing for direction $j$. (See Fig. 1) For non-perpendicular directions 


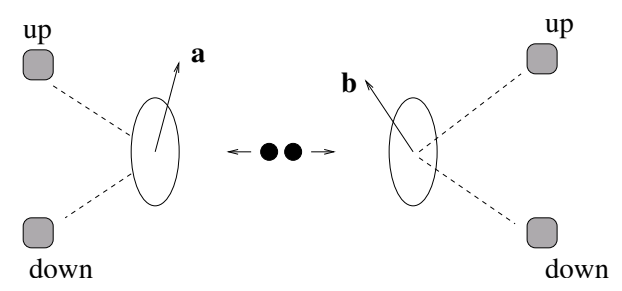

Figure 1: EPR-Bohm setup for spin- $\frac{1}{2}$ particles

$i$ and $j$, respectively quantum mechanics yields correlation between the conditional probabilities via

$$
\begin{array}{r}
p\left(A_{i} B_{j} \mid a_{i} b_{j}\right)=\operatorname{Tr}\left(W_{\left|\Psi_{s}\right\rangle} P_{A_{i}} \otimes P_{B_{j}}\right)=\frac{1}{2} \sin ^{2}\left(\frac{\theta_{a_{i} b_{j}}}{2}\right) \\
p\left(A_{i} \mid a_{i} b_{j}\right)=\operatorname{Tr}\left(W_{\left|\Psi_{s}\right\rangle} P_{A_{i}} \otimes I\right)=\frac{1}{2} \\
p\left(B_{j} \mid a_{i} b_{j}\right)=\operatorname{Tr}\left(W_{\left|\Psi_{s}\right\rangle} I \otimes P_{B_{j}}\right)=\frac{1}{2}
\end{array}
$$

where $W_{\left|\Psi_{s}\right\rangle}$ is the density operator pertaining to the pure state $\left|\Psi_{s}\right\rangle ; P_{A_{i}}$ and $P_{B_{j}}$ denote projections on the eigensubspaces with eigenvalue +1 of the spin operators associated with directions $a_{i}$ and $b_{j}$ respectively; and $\theta_{a_{i} b_{j}}$ denotes the angle between directions $a_{i}$ and $b_{j}$. Specially, if $a_{i}$ and $b_{j}$ are parallel directions then there is a perfect anticorrelation between the outcomes via

$$
p\left(A_{i} \bar{B}_{j} \mid a_{i} b_{j}\right)=p\left(A_{i} \mid a_{i} b_{j}\right)=p\left(\bar{B}_{j} \mid a_{i} b_{j}\right)=\frac{1}{2}
$$

Now, a full-fledged common-causal explanation of the EPR correlations must comply with three demands on the statistical level. Firstly, all the correlations must be screened-off by a common common cause system. (Recall that the definition of the common cause system was the weakest among the four different definitions of the common cause.) Secondly, statistical relations among the measurement outcomes and the measurement settings must reflect the spacetime location of these events in the sense that spatially separated events have to be statistically independent. Thirdly, measurement settings should not be influenced by the common common cause system that is the measurement settings and the common common cause system also have to be statistically independent. We refer to these requirements in turn as common screener-off system, locality and no-conspiracy. The precise probabilistic formulation of these demands is the following:

1. Common screener-off system: There exists a classical probability measure space $(\Omega, p)$ and a $\left\{C_{k}\right\}_{k=1}^{n}$ partition of $\Omega$ such that for every $A_{i}, B_{j}, a_{i}$ and $b_{j}$ in $\Omega(i, j=1,2,3 ; k=1 \ldots n)$ the following factorization holds:

$$
p\left(A_{i} B_{j} \mid a_{i} b_{j} C_{k}\right)=p\left(A_{i} \mid a_{i} b_{j} C_{k}\right) p\left(B_{j} \mid a_{i} b_{j} C_{k}\right)
$$

2. Locality: For every $A_{i}, B_{j}, a_{i}, b_{j}$ and $C_{k}$ in $\Omega(i, j=1,2,3 ; k=1 \ldots n)$ the following screeningoff holds:

$$
p\left(A_{i} \mid a_{i} b_{j} C_{k}\right)=p\left(A_{i} \mid a_{i} C_{k}\right) \quad p\left(B_{j} \mid a_{i} b_{j} C_{k}\right)=p\left(B_{j} \mid b_{j} C_{k}\right)
$$

3. No-conspiracy: Consider the algebra $\mathfrak{D} \subset \Omega$ consisting of the $C_{k}$ elements in the partition $\left\{C_{k}\right\}_{k=1}^{n}$ and all the possible disjunctions thereof. Then for any element $E \in \mathfrak{D}$ and $a_{i}, b_{j}$ in 
$\Omega(i, j=1,2,3)$ the following independence holds: ${ }^{1}$

$$
p\left(a_{i} b_{j} E\right)=p\left(a_{i} b_{j}\right) p(E)
$$

In standard common-causal explanations one usually demands instead of (22) that for every $a_{i}, b_{j}$, and $C_{k}$ in $\Omega(i, j=1,2,3 ; k=1 \ldots n)$ the following independence should hold:

$$
p\left(a_{i} b_{j} C_{k}\right)=p\left(a_{i} b_{j}\right) p\left(C_{k}\right)
$$

It is straightforward to see that (23) entails (22) that is the independence of the elements of the common common cause system from the choice of the measurement settings assures that any disjunction of the elements of the common common cause system will also be independence of the choice of the measurement settings. Thus, in the case of the common-common-cause-system-type explanations equation (23) will suffice as a no-conspiracy requirement.

However, as it is well-known (20), (21), and (23) result in various Bell inequalities which are violated for special measurement settings in the EPR experiment. Consequently, EPR correlations fall short of local, non-conspiratorial, common-common-cause-system-type explanation. One premise has to be given up.

\section{Szabó's separate-common-cause-models for the EPR corre- lations}

As it was noted in the Introduction Szabó's idea was to replace the concept of common common cause system with that of separate common causes and to provide a local, non-conspiratorial, separatecommon-cause-explanation for the EPR. A separate-common-cause-explanation for the EPR consists in finding nine ${ }^{2} C_{i j}$ events $(i, j=1,2,3)$ such that screening-off, locality, and no-conspiracy hold in the following sense:

1. Separate screener-offs: There exists a classical probability measure space $(\Omega, p)$ and nine $C_{i j}$ events in $\Omega$ such that for every $A_{i}, B_{j}, a_{i}, b_{j}(i, j=1,2,3)$ the following factorizations hold:

$$
\begin{aligned}
p\left(A_{i} B_{j} \mid a_{i} b_{j} C_{i j}\right) & =p\left(A_{i} \mid a_{i} b_{j} C_{i j}\right) p\left(B_{j} \mid a_{i} b_{j} C_{i j}\right) \\
p\left(A_{i} B_{j} \mid a_{i} b_{j} \bar{C}_{i j}\right) & =p\left(A_{i} \mid a_{i} b_{j} \bar{C}_{i j}\right) p\left(B_{j} \mid a_{i} b_{j} \bar{C}_{i j}\right)
\end{aligned}
$$

2. Locality: For every $A_{i}, B_{j}, a_{i}, b_{j}(i, j=1,2,3)$ and the appropriate $C_{i j}$ in $\Omega$ the following screening-offs hold:

$$
\begin{array}{cc}
p\left(A_{i} \mid a_{i} b_{j} C_{i j}\right)=p\left(A_{i} \mid a_{i} C_{i j}\right), & p\left(B_{j} \mid a_{i} b_{j} C_{i j}\right)=p\left(B_{j} \mid b_{j} C_{i j}\right) \\
p\left(A_{i} \mid a_{i} b_{j} \bar{C}_{i j}\right)=p\left(A_{i} \mid a_{i} \bar{C}_{i j}\right), & p\left(B_{j} \mid a_{i} b_{j} \bar{C}_{i j}\right)=p\left(B_{j} \mid b_{j} \bar{C}_{i j}\right)
\end{array}
$$

3. No-conspiracy: Consider the algebra $\mathfrak{C} \subset \Omega$ consisting of the separate common causes $C_{i j}$ in $\Omega$ $(i, j=1,2,3)$ and all the possible conjunctions and disjunctions thereof. Then for any element $F \in \mathfrak{C}$ and $a_{i}, b_{j}$ in $\Omega(i, j=1,2,3)$ the following independence holds:

$$
p\left(a_{i} b_{j} F\right)=p\left(a_{i} b_{j}\right) p(F)
$$

\footnotetext{
${ }^{1}$ For a full no-conspiracy one should require the independence of the choice of the measurement settings from the conjunctions of any element of $\mathfrak{D}$ and any other elements in $\Omega$ representing a physically independent event from the choice of the measurement settings. However, for our purposes (22) will suffice.

${ }^{2}$ In (2000) Szabó used the $2 \times 2$ version of the EPR scenario with $i, j=1,2$ hence the number of the separate common causes was four.
} 
Now, consider again the 'reduced' version of no-conspiracy similar to the common-common-causesystem-type case in the last Section. Assume that for every $a_{i}, b_{j}$ and $C_{k l}$ in $\Omega(i, j, k, l=1,2,3)$ the following independence holds:

$$
p\left(a_{i} b_{j} C_{k l}\right)=p\left(a_{i} b_{j}\right) p\left(C_{k l}\right)
$$

Here, requirement (29) does not entail (28) that is the independence of the separate common causes from the choice of the measurement settings does not assure that any combination of the separate causes and their complement will also be independent from the choice of the measurement settings. Thus, in the case of separate-common-cause-system-type explanations (29) does not regard as a sufficient no-conspiracy requirement.

The difference between requirements (28) and (29) is nicely illustrated in (Szabó, 2000). Szabó provided a number of separate-common-cause-models for the EPR situation such that the models were local and non-conspiratorial in the sense of (29). However, the models were conspiratorial in the sense of (28). As Szabó puts it, "combinations of the common cause events as $C_{i j} C_{i j^{\prime}}, C_{i j} \cup C_{i j^{\prime}}$, $C_{i j} C_{i j^{\prime}} C_{i^{\prime} j}$ etc. do statistically correlate with the measurement operations" (Szabó, 2000). Finally, after numerous computer simulations aiming to remove the unwanted conspiracies Szabó concluded with the conjecture that EPR can not be given any local, separate-common-cause-model free from all type of conspiracies.

\section{The separate-common-causes-type derivation of the Bell in- equalities by Graßhoff et al.}

Szabó's conjecture was proved by Graßhoff, Portmann, and Wüthrich in the course of their project initiated in 2000. As they write it in (Graßhoff et al., 2005, p. 668): "Whether a model can be constructed without these correlations [conspiracies] was posed as an open question by Szabó. This question is answered negatively by the derivation of Bell's inequalities." Having seen Szabó's numerous conspiratorial separate-common-cause-models for the EPR correlations Graßhoff et al. turned the problem upside-down and derived the Bell inequalities from the same premises. The details of the derivation are the following:

Consider three pairs of (conditionally) perfectly anticorrelating events $\left(A_{i}, \bar{B}_{i}\right)(i=1,2,3)$ in $\Omega$ that is

$$
p\left(A_{i} \bar{B}_{i} \mid a_{i} b_{i}\right)=p\left(A_{i} \mid a_{i} b_{i}\right)=p\left(\bar{B}_{i} \mid a_{i} b_{i}\right)
$$

where $a_{i}$ and $b_{j}$ in $\Omega$ represent the choice of the measurement settings as above. Suppose that each correlating pair possesses a separate common cause ${ }^{3} C_{i}$ that is

$$
\begin{aligned}
& p\left(A_{i} \bar{B}_{i} \mid a_{i} b_{i} C_{i}\right)=p\left(A_{i} \mid a_{i} b_{i} C_{i}\right) p\left(\bar{B}_{i} \mid a_{i} b_{i} C_{i}\right) \\
& p\left(A_{i} \bar{B}_{i} \mid a_{i} b_{i} \bar{C}_{i}\right)=p\left(A_{i} \mid a_{i} b_{i} \bar{C}_{i}\right) p\left(\bar{B}_{i} \mid a_{i} b_{i} \bar{C}_{i}\right)
\end{aligned}
$$

holds for $i=1,2,3$. From assumptions (30)-(32) the deterministic nature of the causes follows that is for all $i=1,2,3$

$$
\begin{aligned}
p\left(A_{i} \mid a_{i} b_{i} C_{i}\right) & =p\left(\bar{B}_{i} \mid a_{i} b_{i} C_{i}\right)=1 \\
p\left(A_{i} \mid a_{i} b_{i} \bar{C}_{i}\right) & =p\left(\bar{B}_{i} \mid a_{i} b_{i} \bar{C}_{i}\right)=0
\end{aligned}
$$

Furthermore, assume that each common cause $C_{i}(i=1,2,3)$ is local in the following sense:

$$
\begin{array}{rlrl}
p\left(A_{i} \mid a_{i} b_{i} C_{i}\right) & =p\left(A_{i} \mid a_{i} C_{i}\right), & & p\left(\bar{B}_{i} \mid a_{i} b_{i} C_{i}\right)=p\left(\bar{B}_{i} \mid b_{i} C_{i}\right) \\
p\left(A_{i} \mid a_{i} b_{i} \bar{C}_{i}\right)=p\left(A_{i} \mid a_{i} \bar{C}_{i}\right), & & p\left(\bar{B}_{i} \mid a_{i} b_{i} \bar{C}_{i}\right)=p\left(\bar{B}_{i} \mid b_{i} \bar{C}_{i}\right)
\end{array}
$$

\footnotetext{
${ }^{3}$ In (Graßhoff et al., 2005) the authors start from separate common cause systems instead of separate common causes. Then they point out that in the case of perfect correlations the former reduces to the latter.
} 
Requirements (35)-(36) transform (33)-(34) into

$$
\begin{aligned}
p\left(A_{i} \mid a_{i} C_{i}\right) & =p\left(\bar{B}_{i} \mid b_{i} C_{i}\right)=1 \\
p\left(A_{i} \mid a_{i} \bar{C}_{i}\right) & =p\left(\bar{B}_{i} \mid b_{i} \bar{C}_{i}\right)=0
\end{aligned}
$$

Finally, assume that for all $i, j=1,2,3 ; i \neq j$ the following type of no-conspiracy holds:

$$
p\left(a_{i} b_{j} C_{i} \bar{C}_{j}\right)=p\left(a_{i} b_{j}\right) p\left(C_{i} \bar{C}_{j}\right)
$$

Now, from (37)-(39) one obtains for $i, j=1,2,3 ; i \neq j$

$$
p\left(A_{i} B_{j} \mid a_{i} b_{j}\right)=p\left(C_{i} \bar{C}_{j}\right)
$$

Using the theorem of total probability and some natural assumptions spelling out the probabilistic feature of the model Graßhoff et al. arrive at the following (Wigner type) Bell inequalities

$$
p\left(A_{i} B_{j} \mid a_{i} b_{j}\right) \leqslant p\left(A_{i} B_{k} \mid a_{i} b_{k}\right)+p\left(A_{k} B_{j} \mid a_{k} b_{j}\right)
$$

where $i, j, k=1,2,3$ and $i \neq j, j \neq k, k \neq i$.

This derivation of the Bell inequalities from separate common causes proves Szabó's conjecture. Recall that Szabó's conjecture was that there existed no local, non-conspiratorial, separate-commoncause-model for the EPR correlations. Now, consider the EPR situation with the co-planar setting $\theta_{a_{1} b_{2}}=\frac{\pi}{4}, \theta_{a_{2} b_{3}}=\frac{\pi}{2}, \theta_{a_{1} b_{3}}=\frac{3 \pi}{4}, \theta_{a_{i} b_{i}}=0$ where $a_{i}$ and $b_{i}(i=1,2,3)$ are parallel measuring directions in the opposite wings. According to quantum mechanics, measuring in parallel directions yields perfect anticorrelation between the outcomes via (19) and hence the assumption (30) of the derivation by Graßhoff et al. holds. Now, any local, non-conspiratorial, separate-common-causemodel for these perfectly anticorrelating pairs of events results in the Bell inequalities (41). However, for the above measuring directions the conditional probabilities yield

$$
\begin{aligned}
& p\left(A_{1} B_{2} \mid a_{1} b_{2}\right)=\frac{2-\sqrt{2}}{8} \\
& p\left(A_{2} B_{3} \mid a_{2} b_{3}\right)=\frac{1}{4} \\
& p\left(A_{1} B_{3} \mid a_{1} b_{3}\right)=\frac{2+\sqrt{2}}{8}
\end{aligned}
$$

for which the Bell inequalities (41) are maximally violated. Thus, Szabó's conjecture that there exists no local, non-conspiratorial, separate-common-cause-model for all EPR correlations is proven.

\section{Separate- versus common-common-cause-type derivations of the Bell inequalities}

At the end of the paper Graßhoff, Portmann, and Wüthrich claim the following: "The derivation of the Bell inequality presented here is an improvement on the usual Bell-type arguments ... it does not assume a common common cause variable [system] for different correlations" (Graßhoff et al., 2005, p. 676). Although this claim is true, it does not mean that the separate-common-cause-type derivation of the Bell inequalities presented by Graßhoff et al. has no connection to a common-common-causesystem-type derivation. In this Section it will be argued that the assumptions of the above separatecommon-cause-type derivation entail the mathematical assumptions of the usual common-commoncause-system-type derivation. We achieve this by explicitly constructing the common screener-off system which underlies the above separate screener-offs. 
Consider the 2-partitions $\left\{C_{i}, \bar{C}_{i}\right\}$ in (31)-(32) where $i=1,2,3$ and combine them in a finer $n$-partition according to

$$
\left\{D^{\varepsilon}\right\}_{\varepsilon \in\{0,1\}^{3}} \equiv \bigcap_{i=1}^{3} C_{i}^{\varepsilon_{i}}
$$

where $\varepsilon_{i} \in\{0,1\}$ for all $i=1,2,3$, and $C_{i}^{\varepsilon_{i}}=C_{i}$ if $\varepsilon_{i}=1$ and $C_{i}^{\varepsilon_{i}}=\bar{C}_{i}$ if $\varepsilon_{i}=0$. (Obviously, $\left\{D^{\varepsilon}\right\}_{\varepsilon \in\{0,1\}^{3}}$ is maximally an 8-partition.) The elements of $\left\{D^{\varepsilon}\right\}_{\varepsilon \in\{0,1\}^{3}}$ are the logical atoms of the algebra generated by the 2-partitions $\left\{C_{i}, \bar{C}_{i}\right\}(i=1,2,3)$.

Now, we prove in turn that the partition $\left\{D^{\varepsilon}\right\}_{\varepsilon \in\{0,1\}^{3}}$ screens all correlations (30) off, it is local, and non-conspiratorial.

1. Screening-off. Since the anticorrelation between events $\left(A_{i}, \bar{B}_{i}\right)$ in $\Omega(i=1,2,3)$ is perfect, the appropriate separate common causes are deterministic in the sense of (33)-(34). As for all $\varepsilon \in\{0,1\}^{3}$ there exists an $i=1,2,3$ such that $D^{\varepsilon} \subseteq C_{i}$ or $D^{\varepsilon} \subseteq \bar{C}_{i}$ therefore for all $\varepsilon \in\{0,1\}^{3}$

$$
p\left(A_{i} \mid a_{i} b_{i} D^{\varepsilon}\right)=p\left(\bar{B}_{i} \mid a_{i} b_{i} D^{\varepsilon}\right) \in\{0,1\}
$$

and hence for all $\varepsilon \in\{0,1\}^{3}$

$$
p\left(A_{i} \bar{B}_{i} \mid a_{i} b_{i} D^{\varepsilon}\right)=p\left(A_{i} \mid a_{i} b_{i} D^{\varepsilon}\right) p\left(\bar{B}_{i} \mid a_{i} b_{i} D^{\varepsilon}\right)
$$

which means that $\left\{D^{\varepsilon}\right\}_{\varepsilon \in\{0,1\}^{3}}$ is a (deterministic) common screener-off system of all three correlations (30).

2. Locality. Again, since for all $\varepsilon \in\{0,1\}^{3}$ there exists an $i=1,2,3$ such that $D^{\varepsilon} \subseteq C_{i}$ or $D^{\varepsilon} \subseteq \bar{C}_{i}$ therefore from (35)-(36) it follows that for all $\varepsilon \in\{0,1\}^{3}$

$$
p\left(A_{i} \mid a_{i} b_{i} D^{\varepsilon}\right)=p\left(A_{i} \mid a_{i} D^{\varepsilon}\right) p\left(\bar{B}_{i} \mid a_{i} b_{i} D^{\varepsilon}\right)=p\left(\bar{B}_{i} \mid b_{i} D^{\varepsilon}\right)
$$

which means that the common screener-off system $\left\{D^{\varepsilon}\right\}_{\varepsilon \in\{0,1\}^{3}}$ is local.

3. No-conspiracy. Recall that the aim of Graßhoff et al. was to prove Szabó's conjecture that there existed no local, separate-common-cause-model for the EPR which is free from all type of conspiracies in the sense of (28). To achieve this goal, it is enough to derive Bell inequalities from the no-conspiracy requirement of any element $F$ of the generated algebra $\mathfrak{C}$ by the separate common causes. They picked out the elements $C_{i} \bar{C}_{j}(i, j=1,2,3 ; i \neq j)$ and required no-conspiracy in form (39). The simple reason why Graßhoff et al. employed $C_{i} \bar{C}_{j}$ in the no-conspiracy requirement was that in the derivation of the Bell inequalities (41) it was the combined term $C_{i} \bar{C}_{j}(i, j=1,2,3 ; i \neq j)$ that was needed.

Now, no-conspiracy (39) can be translated into the common-common-cause-screener-off-model $\left\{D^{\varepsilon}\right\}_{\varepsilon \in\{0,1\}^{3}}$ as follows. Consider the algebra $\mathfrak{C}$ generated by the separate screener-offs $C_{i}(i=$ $1,2,3)$ and the algebra $\mathfrak{D}$ generated by the common screener-off system $\left\{D^{\varepsilon}\right\}_{\varepsilon \in\{0,1\}^{3}}$, respectively. Regarding relation (45) it is straightforward to verify that the two algebras $\mathfrak{C}$ and $\mathfrak{D}$ are identical. Since the elements $C_{i} \bar{C}_{j}(i, j=1,2,3 ; i \neq j)$ in $(39)$ are identical with the disjunctions $D^{i \bar{j}} \equiv$ $D^{i \bar{j} k} \cup D^{i \bar{j} k}$ where $D^{i \bar{j} k}=C_{i} \bar{C}_{j} C_{k}$ and $D^{i \overline{j k}}=C_{i} \bar{C}_{j} \bar{C}_{k}$ therefore no-conspiracy (39) comes to be equivalent to

$$
p\left(a_{i} b_{j} D^{i \bar{j}}\right)=p\left(a_{i} b_{j}\right) p\left(D^{i \bar{j}}\right)
$$

Thus, requirement (49) prescribes the same no-conspiracy for the common screener-off system $\left\{D^{\varepsilon}\right\}_{\varepsilon \in\{0,1\}^{3}}$ as requirement (39) prescribes for the separate screener-offs. 
To sum up, assuming perfectly anticorrelating pairs of events the separate-common-screener-offmodel of Graßhoff et al. becomes identical with a common-screener-off-system-model via relation (45) in the following way. Screening-off relations (31)-(32) of the separate screener-offs are identical with screening-off relation (47) of the common screener-off system. Locality (35)-(36) of the separate screener-offs is equivalent to locality (48) of the common screener-off system. No-conspiracy requirement (39) for some special conjunctions of the separate screener-offs is identical with no-conspiracy (49) for some special disjunctions of the elements of the common screener-off system.

Now, according to the derivation of Graßhoff et al. requirements (31)-(39) together entail Bell inequalities (41). Consequently, the equivalent assumptions (47), (48) and (49), respectively will entail the same Bell inequalities. Regarding this fact, the separate-common-screener-off-type derivation of Graßhoff et al. is reducible to a common-screener-off-system-type derivation.

Expressing the above results in the 'common cause' terminology instead of the 'screener-off' terminology the situation is the following. Since the notion of common cause is stronger then that of the appropriate screener-off, we are not allowed to interpret our result as a reduction of the set of the local, non-conspiratorial separate common causes to a local, non-conspiratorial common common cause(!) system. All we can say is that we reduced the set of the local, non-conspiratorial separate common causes of Graßhoff et al. to a local, non-conspiratorial common screener-off(!) system which is not necessarily a common common cause system. Notice however that standard common-commoncause-system-type derivations of the Bell inequalities make use of no other properties of the common common cause system than screening-off, locality and no-conspiracy. In this sense our reduction reproduces all the essential assumptions of a common-common-cause-system-type derivation of the Bell inequalities.

\section{A non-reducible separate-common-cause-type derivation of the Bell inequalities}

The derivation of Graßhoff et al. crucially depended on the fact that the correlations were perfect. If requirement (30) is dropped the Bell inequalities (41) can not be derived the way they have done. This fact leaves open the question whether any set of non-perfect EPR correlations can be given a local, non-conspiratorial, separate-common-cause-model. Graßhoff et al. (2005, p. 677) put it as follows: "We have not been able to derive a Bell-type inequality ruling out perfect correlations and allowing different common cause variables [separate common causes]. If PCORR [perfect correlation] is indeed a necessary assumption for our derivation of the Bell inequality, it should be possible to construct a model in which PCORR [perfect correlation] does not hold (being violated by arbitrary small deviation, say)."

In other words, the derivation of the Bell inequalities by Graßhoff et al. from perfectly correlating pairs of events does not exclude the possibility of a separate-common-cause-explanation of the non-perfect EPR correlations. Since perfect correlations can not be tested experimentally with absolute precision, the derivation of the Bell inequalities from perfect correlations does not provide an experimentally verifiable refutation of separate-common-cause-models for the EPR. In the following we close this experimental loophole of the project of Graßhoff et al. by presenting a separate-common-cause-derivation of some Bell-like inequalities from non-perfect correlations.

Consider three pairs of (conditionally) correlating events $\left(A_{i}, \bar{B}_{i}\right)(i=1,2,3)$ in $\Omega$ and assume that each correlating pair possesses a separate common cause $C_{i}$, that is

$$
\begin{aligned}
& p\left(A_{i} \bar{B}_{i} \mid a_{i} b_{i} C_{i}\right)=p\left(A_{i} \mid a_{i} b_{i} C_{i}\right) p\left(\bar{B}_{i} \mid a_{i} b_{i} C_{i}\right) \\
& p\left(A_{i} \bar{B}_{i} \mid a_{i} b_{i} \bar{C}_{i}\right)=p\left(A_{i} \mid a_{i} b_{i} \bar{C}_{i}\right) p\left(\bar{B}_{i} \mid a_{i} b_{i} \bar{C}_{i}\right)
\end{aligned}
$$

holds for $i=1,2,3$. Furthermore, assume that each common cause $C_{i}(i=1,2,3)$ is local in the 
following sense:

$$
\begin{array}{cc}
p\left(A_{i} \mid a_{i} b_{i} C_{i}\right)=p\left(A_{i} \mid a_{i} C_{i}\right), & p\left(\bar{B}_{i} \mid a_{i} b_{i} C_{i}\right)=p\left(\bar{B}_{i} \mid b_{i} C_{i}\right) \\
p\left(A_{i} \mid a_{i} b_{i} \bar{C}_{i}\right)=p\left(A_{i} \mid a_{i} \bar{C}_{i}\right), & p\left(\bar{B}_{i} \mid a_{i} b_{i} \bar{C}_{i}\right)=p\left(\bar{B}_{i} \mid b_{i} \bar{C}_{i}\right)
\end{array}
$$

Let us denote the conditional probabilities by

$$
\begin{aligned}
& p\left(A_{i} \mid a_{i} C_{i}\right)=1-\gamma_{i}^{A} \\
& p\left(\bar{B}_{i} \mid b_{i} C_{i}\right)=1-\gamma_{i}^{B} \\
& p\left(A_{i} \mid a_{i} \bar{C}_{i}\right)=\bar{\gamma}_{i}^{A} \\
& p\left(\bar{B}_{i} \mid b_{i} \bar{C}_{i}\right)=\bar{\gamma}_{i}^{B}
\end{aligned}
$$

Finally, assume that for all $i, j=1,2,3 ; i \neq j$ the following type of no-conspiracy holds:

$$
p\left(a_{i} b_{j} C_{i} \bar{C}_{j}\right)=p\left(a_{i} b_{j}\right) p\left(C_{i} \bar{C}_{j}\right)
$$

Now, from (50)-(58) one obtains the following bounds for the conditional probabilities $(i, j=1,2,3$; $i \neq j)$ :

$$
\begin{aligned}
p\left(A_{i} B_{j} \mid a_{i} b_{j}\right) \geqslant & \left(1-\gamma_{i}^{A} \frac{p\left(a_{i} C_{i}\right)}{p\left(a_{i} b_{j} C_{i} \bar{C}_{j}\right)}-\bar{\gamma}_{j}^{B} \frac{p\left(b_{j} \bar{C}_{j}\right)}{p\left(a_{i} b_{j} C_{i} \bar{C}_{j}\right)}\right) p\left(C_{i} \bar{C}_{j}\right) \\
& +\left(\bar{\gamma}_{i}^{A} \frac{p\left(a_{i} \bar{C}_{i}\right)}{p\left(a_{i} b_{j}\left(\bar{C}_{i} \cup C_{j}\right)\right)}+\gamma_{j}^{B} \frac{p\left(b_{j} C_{j}\right)}{p\left(a_{i} b_{j}\left(\bar{C}_{i} \cup C_{j}\right)\right)}\right) p\left(\bar{C}_{i} \cup C_{j}\right) \\
p\left(A_{i} B_{j} \mid a_{i} b_{j}\right) \leqslant & p\left(C_{i} \bar{C}_{j}\right)+\left(\bar{\gamma}_{i}^{A} \frac{p\left(a_{i} \bar{C}_{i}\right)}{p\left(a_{i} b_{j}\left(\bar{C}_{i} \cup C_{j}\right)\right)}+\gamma_{j}^{B} \frac{p\left(b_{j} C_{j}\right)}{p\left(a_{i} b_{j}\left(\bar{C}_{i} \cup C_{j}\right)\right)}\right) p\left(\bar{C}_{i} \cup C_{j}\right)
\end{aligned}
$$

Using the theorem of total probability after some tedious but trivial calculation one arrives at the following Bell-like inequalities

$$
p\left(A_{i} B_{j} \mid a_{i} b_{j}\right)+f_{i j}^{k} \leqslant p\left(A_{i} B_{k} \mid a_{i} b_{k}\right)+p\left(A_{k} B_{j} \mid a_{k} b_{j}\right)
$$

where $i, j, k=1,2,3$ and $i \neq j, j \neq k, k \neq i$ and the functions $f_{i j}^{k}$ are the following:

$$
\begin{aligned}
f_{i j}^{k}= & \left(\bar{\gamma}_{i}^{A} \frac{p\left(a_{i} \bar{C}_{i}\right)}{p\left(a_{i} b_{k}\left(\bar{C}_{i} \cup C_{k}\right)\right)}+\gamma_{k}^{B} \frac{p\left(b_{k} C_{k}\right)}{p\left(a_{i} b_{k}\left(\bar{C}_{i} \cup C_{k}\right)\right)}\right) p\left(\bar{C}_{i} \cup C_{k}\right) \\
& +\left(\bar{\gamma}_{k}^{A} \frac{p\left(a_{k} \bar{C}_{k}\right)}{p\left(a_{k} b_{j}\left(\bar{C}_{k} \cup C_{j}\right)\right)}+\gamma_{j}^{B} \frac{p\left(b_{j} C_{j}\right)}{p\left(a_{k} b_{j}\left(\bar{C}_{k} \cup C_{j}\right)\right)}\right) p\left(\bar{C}_{k} \cup C_{j}\right) \\
& -\left(\bar{\gamma}_{i}^{A} \frac{p\left(a_{i} \bar{C}_{i}\right)}{p\left(a_{i} b_{j}\left(\bar{C}_{i} \cup C_{j}\right)\right)}+\gamma_{j}^{B} \frac{p\left(b_{j} C_{j}\right)}{p\left(a_{i} b_{j}\left(\bar{C}_{i} \cup C_{j}\right)\right)}\right) p\left(\bar{C}_{i} \cup C_{j}\right) \\
& -\left(\gamma_{i}^{A} \frac{p\left(a_{i} C_{i}\right)}{p\left(a_{i} b_{k} C_{i} \bar{C}_{k}\right)}+\bar{\gamma}_{k}^{B} \frac{p\left(b_{k} \bar{C}_{k}\right)}{p\left(a_{i} b_{k} C_{i} \bar{C}_{k}\right)}\right) p\left(C_{i} \bar{C}_{k}\right) \\
& -\left(\gamma_{k}^{A} \frac{p\left(a_{k} C_{k}\right)}{p\left(a_{k} b_{j} C_{k} \bar{C}_{j}\right)}+\bar{\gamma}_{j}^{B} \frac{p\left(b_{j} \bar{C}_{j}\right)}{p\left(a_{k} b_{j} C_{k} \bar{C}_{j}\right)}\right) p\left(C_{k} \bar{C}_{j}\right)
\end{aligned}
$$

Now, let us introduce a so-called 'deviation parameter' which measures the maximum deviation of a set of correlations from perfect anticorrelation. In other words, let $\delta \in[0,1]$ be a real number such that

$$
\delta=\max _{i}\left\{p\left(A_{i} \mid a_{i} b_{i}\right)-p\left(A_{i} \bar{B}_{i} \mid a_{i} b_{i}\right) ; p\left(\bar{B}_{i} \mid a_{i} b_{i}\right)-p\left(A_{i} \bar{B}_{i} \mid a_{i} b_{i}\right)\right\}
$$


It is straightforward to see that from assumptions (54)-(57) and (62) it follows that for all $i=1,2,3$

$$
\begin{aligned}
& \frac{1}{2}-\sqrt{\frac{1}{4}-\frac{\delta}{p\left(C_{i}\right)}} \leqslant \gamma_{i}^{A}, \gamma_{i}^{B} \leqslant \frac{1}{2}+\sqrt{\frac{1}{4}-\frac{\delta}{p\left(C_{i}\right)}} \\
& \frac{1}{2}-\sqrt{\frac{1}{4}+\frac{\delta}{p\left(\bar{C}_{i}\right)}} \leqslant \bar{\gamma}_{i}^{A}, \bar{\gamma}_{i}^{B} \leqslant \frac{1}{2}+\sqrt{\frac{1}{4}+\frac{\delta}{p\left(\bar{C}_{i}\right)}}
\end{aligned}
$$

and hence all $\gamma_{i}^{A}, \gamma_{i}^{B}, \bar{\gamma}_{i}^{A}$ and $\bar{\gamma}_{i}^{B}$, respectively $(i=1,2,3)$ vanish as $\delta$ tends to 0 . However, with $\gamma_{i}^{A}$, $\gamma_{i}^{B}, \bar{\gamma}_{i}^{A}$ and $\bar{\gamma}_{i}^{B}$ tending to zero the functions $f_{i j}^{k}$ also tend (continuously) to zero and the Bell-like inequalities (61) tend (continuously) to the Bell inequalities (41) derived from perfect anticorrelation. Concisely, the closer the correlations are to perfect anticorrelation, the closer are the derived Bell-like inequalities to the Bell inequalities (41). Let Bell $(\delta)$ denote those Bell-like inequalities that result from a set of correlations of deviation parameter $\delta$.

Now, consider again the EPR situation with the measurement directions $\theta_{a_{1} b_{2}}=\frac{\pi}{4}, \theta_{a_{2} b_{3}}=\frac{\pi}{2}$, $\theta_{a_{1} b_{3}}=\frac{3 \pi}{4}$ and $\theta_{a_{i} b_{i}}=0$, respectively. Because of the parallel measuring directions quantum mechanics predicts a perfect correlation between the events $\left(A_{i}, \bar{B}_{i}\right)(i=1,2,3)$. As stated before, for this setting Bell inequalities are maximally $(\sqrt{2}<1$ !) violated. However, assume now that due to experimental imperfections we are unable to test the perfect anticorrelations with absolute precision and all we know is that the deviation from perfect anticorrelation is less than $\delta$. Denote any set of such correlations by $\operatorname{EPR}(\delta)$. Now, regarding that the $\operatorname{Bell}(\delta)$ inequalities continuously tend with $\delta$ to the maximally violated Bell inequalities (41), there exists a 'deviation threshold' $\sigma \in[0,1]$ such that for any $\operatorname{EPR}(\delta)$ set such that $\delta<\sigma$ the resulting $\operatorname{Bell}(\delta)$ inequalities are violated. This excludes that for arbitrary small deviation from perfect anticorrelation there exists a separate-common-causesystem-model for the EPR.

There exists another interpretation of the $\operatorname{EPR}(\delta)$ set. Suppose we have no limit on experimental precision. Suppose furthermore that the measuring directions are chosen 'very close' to the above parallel settings that is $\theta_{a_{1} a_{2}}=\frac{\pi}{4}, \theta_{a_{2} a_{3}}=\frac{\pi}{2}, \theta_{a_{1} a_{3}}=\frac{3 \pi}{4}$ and $0<\theta_{a_{i} b_{i}}<2 \arcsin \sqrt{2 \sigma}$, where $\sigma$ is the 'deviation threshold'. Then due to (16) the Bell inequalities (61) will be again violated. In other words, assuming absolutely precise experiments there exists a set of measurement directions around the setting $\theta_{a_{1} b_{2}}=\frac{\pi}{4}, \theta_{a_{2} b_{3}}=\frac{\pi}{2}, \theta_{a_{1} b_{3}}=\frac{3 \pi}{4}$ and $\theta_{a_{i} b_{i}}=0$ such that for any measurement direction in this set the Bell inequalities (61) are violated. Thus, there exists no local, non-conspiratorial, separate-common-cause-model for this set.

A very similar result for the Clauser-Horne inequality assuming separate-common-cause-systems was derived by Portmann and Wüthrich (2007), where the authors explicitly calculate the deviation parameter. In our proof we arrive at the Bell inequalities without calculating the deviation parameter, simply exploiting the fact that the conditional probabilities are continuous.

One final point has to be stressed regarding the above derivation. The equivalence of the separate-common-screener-off-derivation and the appropriate common-common-cause-screener-offtype derivation offered in the previous Section depended crucially on the fact that the correlations were perfect. In the non-perfect case the screening-off equations (31)-(32) does not imply (47) and thus $\left\{D^{\varepsilon}\right\}_{\varepsilon \in\{0,1\}^{3}}$ does not provide a common screener-off system for the correlations. Consequently, derivations of the Bell inequalities from a set of non-perfectly correlating pairs of events is not automatically reducible to common-screener-off-system-type derivations. In other words, in the non-perfect case Bell inequalities do not obtain indirectly due to an underlying common screener-off system but directly due to the separate common causes. ${ }^{4}$

\footnotetext{
${ }^{4}$ Obviously, other common screener-off systems might exist for the given set of correlations but they bear no logical relation to the original separate common causes via (45). The general question whether there exist separatecommon-cause-derivations of Bell-like inequalities with no (underlying) common common cause systems is open. (See (Portmann and Wüthrich, 2007)).
} 


\section{Conclusions}

In the paper we have shown the following. The local, non-conspriratorial separate-common-causetype derivation of the Bell inequalities by Graßhoff et al. based on perfect anticorrelations is reducible to a local, non-conspiratorial common-screener-off-system-type derivation. However, not assuming perfect anticorrelations a non-reducible separate-common-cause-type derivation of some $\operatorname{Bell}(\delta)$ inequalities can be presented. The violation of these Bell $(\delta)$ inequalities proves Szabó's (2000) conjecture concerning the non-existence of a local, non-conspiratorial, separate-common-cause-model for an $\operatorname{EPR}(\delta)$ set of correlations.

Acknowledgement. Work supported by János Bolyai Research Scholarship of the Hungarian Academy of Science.

\section{References}

Belnap, N., L. E. Szabó (1996). "Branching Space-Time Analysis of the GHZ Theorem," Foundations of Physics, 26, 982-1002.

Graßhoff, G., S. Portmann, A. Wüthrich (2005). "Minimal Assumption Derivation of a Bell-type Inequality," The British Journal for the Philosophy of Science, 56, 663-680.

Hofer-Szabó, G., M. Rédei, L. E. Szabó (1999). "On Reichenbach's Common Cause Principle and on Reichenbach's Notion of Common Cause," The British Journal for the Philosophy of Science, 50, 377-399.

Hofer-Szabó, G., M. Rédei, L. E. Szabó (2002). "Common Causes are not Common Common Causes," Philosophy of Science, 69, 623-633.

Hofer-Szabó, G., M. Rédei (2004). "Reichenbachian Common Cause Systems," International Journal of Theoretical Physics, 34, 1819-1826.

Hofer-Szabó, G., M. Rédei (2006). "Reichenbachian Common Cause Systems of Arbitrary Finite Size Exist," Foundations of Physics Letters, 35, 745-746.

Placek, T. (2000). Is Nature deterministic?, Jagiellonian University Press, Kraków.

Portmann S., A. Wüthrich (2007). "Minimal Assumption Derivation of a Weak Clauser-Horne Inequality," Studies in History and Philosophy of Modern Physics, (forthcomming), electronic preprint $<$ www.arxiv.org/quant-ph/0604216 $>$.

Reichenbach, H. (1956). The Direction of Time, University of California Press, Berkeley.

Szabó, L. E. (2000). "On an Attempt to Resolve the EPR-Bell Paradox via Reichenbachian Concept of Common Cause," International Journal of Theoretical Physics, 39, 911.

Wüthrich, A. (2004). Quantum Correlations and Common Causes, Master's Thesis, Bern Studies in the History and Philosophy of Science, Bern. 